\title{
Apolipoprotein E polymorphisms contribute to statin response in Chinese ASCVD patients with dyslipidemia
}

\author{
Lei Zhang ${ }^{1}$, Siying $\mathrm{He}^{1}$, Zuhua $\mathrm{Li}^{1}$, Xuedong Gan² , Siwei $\mathrm{Li}^{1}$, Xiaohuan $\mathrm{Cheng}^{1}$, Na Yang ${ }^{1}$ and Fang Zheng ${ }^{1 *}$
}

\begin{abstract}
Background: Apolipoprotein E (ApoE) plays an important role in lipid metabolism and clearance. Statins are the most common drugs used to modulate the lipid profile in the clinic therapy; the associations between ApoE polymorphisms and statin response to lipids were inconsistent in previous studies among different ethnicities. Our study aimed to demonstrate the relationships among the statins response and the ApoE gene common polymorphisms and lifestyle risk factors in Chinese arteriosclerotic cardiovascular disease (ASCVD) patients with dyslipidemia.
\end{abstract}

Methods: A total of 1002 dyslipidemia ASCVD patients were recruited in this study, including 311 patients with a history of type 2 diabetes mellitus (T2DM). These patients were all treated with drugs atorvastatin $(10 \mathrm{mg} / \mathrm{d})$ or rosuvastatin ( $5 \mathrm{mg} / \mathrm{d}$ ) for at least 4 weeks and genotyped for ApoE e2/e3/e4 alleles, using Kompetitive Allele Specific PCR (KASP) and Sanger sequencing. The plasma lipids levels were determined before and after statins treatment.

Results: The results of ApoE genotyping with KASP method were consistent with the sequencing analysis. In the total 1002 patients, the E2 phenotypes (e2/e3, e2/e2) had significant lower low-density lipoprotein cholesterol (LDL-C) baseline levels than subjects with E3 (e3/e3, e2/e4) and E4 (e3/e4, e4/e4) phenotypes ( $P=0.007,0.005$, respectively), and E2 phenotypes had the highest triglyceride (TG) baseline levels. To statins treatment, E2 phenotypes had a better response in TG, Total cholesterol (TC) and LDL-C reduction percentage compared with other phenotypes, and smoking/alcohol drinking status also had a significant influence on statins response of LDL-C lowering. No significant difference was found in the effects of lipids decreasing between atorvastatin and rosuvastatin drugs in all patients.

Conclusions: We developed the KASP technique for the ApoE genotyping, and demonstrated ApoE polymorphisms interacted with smoking/drinking to influence the declining extent of TG, TC and LDL-C levels after statins therapy in Chinese dyslipidemia ASCVD patients. These discoveries developed our cognition with the genetic polymorphisms effects on statin response, which should be taken more seriously in smoking/drinking E4 amino acid isoform carriers.

Keywords: Apolipoprotein E, Polymorphism, Statins response, KASP

\section{Background}

Statins are widely used to reduce the LDL-C of the circulation and improve the lipid profile in hypercholesterolemia and other cardiovascular diseases [1]. However, the effectiveness of statin therapy may be influenced by several factors: treatment adherence, age, gender and even genetic variability of each patient $[2,3]$. Because of this, pharmacogenetic

\footnotetext{
* Correspondence: zhengfang@whu.edu.cn

${ }^{1}$ Center for Gene Diagnosis, Zhongnan Hospital of Wuhan University,

Donghu Road 169\#, Wuhan 430071, China

Full list of author information is available at the end of the article
}

studies of lipid-lowering response to statins were established in investigating the associations between genetic variations and therapeutic response of statins and involved other related processes like metabolism and pharmacokinetics $[4,5]$. Since the association could be partially influenced by the ethnicity of individuals, numbers of patients and the underlying diseases, the previous research didn't always get results in accordance and haven't included lifestyle risk factors as potential confounders.

The Apolipoprotein E (ApoE) gene in human located on the chromosome 19q13.2, the differences of amino

(C) The Author(s). 2019 Open Access This article is distributed under the terms of the Creative Commons Attribution 4.0 International License (http://creativecommons.org/licenses/by/4.0/), which permits unrestricted use, distribution, and 
acid at the location of 112 and 158 caused the three major alleles: e2 (Cys112 and Cys158), e3 (Cys112 and Arg158), e4 (Arg112 and Arg158), because of the two single nucleotide polymorphisms (SNPs) (rs429358 $\mathrm{T}>\mathrm{C}$, rs7412 C > $\mathrm{T})$. The combination of three alleles formed six ApoE genotypes: 3 heterozygous (e2/e3, e2/e4 and e3/e4), and 3 homozygous (e2/e2, e3/e3, e4/e4) [6]. These ApoE gene polymorphisms of individuals affect the lipoproteins clearing and the lipid profile, which is also an important candidate susceptible gene for type2 Diabetes (T2DM) and/or arteriosclerotic cardiovascular disease (ASCVD) [7-10]. But the results of the association between ApoE polymorphisms and the risk of coronary artery disease (CAD) in different populations were conflicted [11-14]. Furthermore, the E4 phenotype has shown the association with the lower response to the statin therapy in some studies [15-17], while there was research reported ApoE polymorphisms showed no significant effect on the response of statin in Chinese patients with hyperlipidemia [18] .

Thus, it is necessary to conduct a study to investigate the effect of statins to lower the lipids profile in the Chinese population carrying different ApoE genotypes and evaluate the interaction on statins response among ApoE variants, living habits and history of diseases.

In addition, though kinds of technique had been applied for ApoE genotyping in the clinic for individual therapy of statin, such as TaqMan assay [19] and high resolution melting (HRM) technique, etc., an efficient and economic genotyping method is required in clinical application [20]. In this research, an efficient and economic KASP technique was successfully established to validate the ApoE polymorphisms, which based on allele-specific primer extension [21] and fluorescencebased genotyping technology.

\section{Methods}

\section{Subjects}

The 1002 patients involved in this study were collected in Zhongnan Hospital of Wuhan University, Central Hospital of Wuhan and Shandong Provincial Hospital from November 2016 to December 2017. All patients in this study were more than 18 years old and with ASCVD. Patients who had the baseline levels of $\mathrm{TC} \geq 3.1 \mathrm{mmol} / \mathrm{L}$ or $\mathrm{LDL}-\mathrm{C} \geq 1.8$ $\mathrm{mmol} / \mathrm{L}$ and have been treated with statins (Atorvastatin 10 $\mathrm{mg} / \mathrm{d}$ or Rosuvastatin $5 \mathrm{mg} / \mathrm{d}$ ) for at least 4 weeks [22, 23], were recruited. In addition, the baseline levels of TG and HDL-C of participants were $1.87(1.25,2.60)$ for TG and $1.09(0.94,1.30)$ for HDL-C. The patients with hepatic, kidney and endocrinological or malignant diseases were excluded.

The informed content was obtained and the study got the approval by the Medical Ethics Committee of Zhongnan Hospital of Wuhan University.

\section{Lipid profiling}

Blood samples were obtained by venipuncture following $12 \mathrm{~h}$ overnight fast. Two measurements of lipids levels were obtained for each sample: prior to treatment and $\geq 4$ weeks post treatment (Atorvastatin $10 \mathrm{mg} / \mathrm{d}$ or Rosuvastatin $5 \mathrm{mg} / \mathrm{d}$ ). The lipids profiles of TG, TC, LDL-C and HDL-C were determined on the automatic clinical chemistry analyzer in the core laboratory. Clinical data of study participants were collected, included age, sex, blood pressure, and histories of smoking, alcohol intake, diabetes mellitus, and lipid-lowering drug treatment (Table 1).

\section{ApoE genotyping}

\section{Sanger sequencing}

Genomic DNA was isolated using the phenol/chloroform method [24]. The ApoE isoforms: E2 (e2/e3, e2/ e2), E3 (e3/e3, e2/e4), E4 (e3e4/, e4/e4) were genotyped in 1002 cases using PCR-Sanger sequencing. The primers for sequencing were described in Table 2. PCR amplification reactions were performed in a final $25 \mu \mathrm{L}$ containing $50 \mathrm{ng}$ of genomic DNA, $5 \mathrm{pM}$ of each primer, $5 \mathrm{mM}$ dNTPs, $0.5 \mu \mathrm{L}$ High-GC polymerase (Gen Star Co. Ltd.), $1 \mu \mathrm{L}$ DMSO, $10 \mu \mathrm{L}$ buffer and $10.5 \mu \mathrm{L}$ sterile deionized water. Amplification was performed in a PCR Cycler GT 9612 (BIO-GENER, Hangzhou, China) following the procedure: $95^{\circ} \mathrm{C} 12 \mathrm{~min}, 94^{\circ} \mathrm{C} 1$ $\min , 72^{\circ} \mathrm{C} 1 \mathrm{~min} ; 95^{\circ} \mathrm{C} 1 \mathrm{~min}, 64.8^{\circ} \mathrm{C} 1 \mathrm{~min}, 72^{\circ} \mathrm{C} 1$ min in 30 cycles; $72^{\circ} \mathrm{C} 10 \mathrm{~min}$. PCR product was directly sequenced in the $3730 \mathrm{XL}$ sequencer (ABI, Thermo Fisher Scientific, Waltham, MA, US).

\section{KASP method}

About 104 cases were randomly chosen for establishing the KASP genotyping method (Fig. 1). The reaction system concluded $50 \mathrm{ng}$ genomic DNA, $5 \mu \mathrm{L}$ master mix (LGC Genomics, Beverly, MA), $0.14 \mu \mathrm{L}$ primers (synthesized in LGC Genomics). KASP primers for each SNP were listed (Table 1). KASP reactions were performed in the CFX-connect (Bio-Rad, US) by the standard protocol (LGC Genomics, Beverly, MA)

Table 1 Clinical characteristics of the study participants

\begin{tabular}{ll}
\hline Characteristics & $N=1002$ \\
\hline Age, years & $65(57-75)$ \\
Male/female & $620 / 382$ \\
Systolic blood pressure $(\mathrm{mmHg})$ & $132(120-145)$ \\
Diastolic blood pressure $(\mathrm{mmHg})$ & $78(70-85)$ \\
Smoking/non-smoking & $303 / 699$ \\
Drinking/non-drinking & $237 / 765$ \\
T2DM/non-T2DM & $311 / 691$ \\
Atorvastatin/Rosuvastatin & $575 / 427$ \\
\hline
\end{tabular}


Table 2 Primers for KASP and Sanger sequencing

\begin{tabular}{ll}
\hline Primers & Primer sequence (5'-3') \\
\hline 1. Sequencing primer-Forward & AACAACTGACCCCGGTGGCG \\
2. Sequencing primer-Reverse & ATGGCGCTGAGGCCGCGCTCGG \\
3. RS429358-primer-FAM & CGCGGACATGGAGGACGTGT \\
4. RS429358-primer-HEX & GCGGACATGGAGGACGTGC \\
5. RS429358-primer-Common & CTCGCCGCGGTACTGCACC \\
6. RS7412-primer-FAM & GATGCCGATGACCTGCAGAAGT \\
7. RS7412-primer-HEX & ATGCCGATGACCTGCAGAAGC \\
8. RS7412-primer-Common & CCCGGCCTGGTACACTGCC
\end{tabular}

Primers "1, 2" for Sanger sequencing, "3, 4, 5" for KASP in rs429358 genotyping, and " $6,7,8$ " for KASP in rs7412 genotyping

with 96-well plate: $94{ }^{\circ} \mathrm{C} 15 \mathrm{~min}$; and then 10 cycles of $94{ }^{\circ} \mathrm{C} 20 \mathrm{~s}, 61^{\circ} \mathrm{C} 60 \mathrm{~s}$ (drop $-0.6^{\circ} \mathrm{C} /$ per cycle); 26 cycles of $94^{\circ} \mathrm{C} 20 \mathrm{~s}$ and $1 \mathrm{~min}$ at $55^{\circ} \mathrm{C}$; at last the read step at $37^{\circ} \mathrm{C}$ for $1 \mathrm{~min}$. Data were analyzed using the Bio-Rad CFX Manager Software (version 3.1).

\section{Statistical analysis}

In order to carry out the association analysis between SNPs and statin therapy, "validness" was set as the LDL-C reduction percentage equaled or greater than $25 \%$, otherwise was "voidness" [25].

Continuous variables with skewed data were expressed as median (M, P25 P75) unless otherwise indicated, normal distribution data was assessed using Kolmogorov-Smirnov test, Mann-Whitney U test was used for comparisons of lipid levels before/after statin treatment. The influences of the ApoE alleles (e2/e3/e4) on the response to statins were assessed by analysis of the Kruskal-Wallis test among three ApoE isoforms. The reduction percentages were calculated as:

(baseline lipids leve-after statin therapy lipids level)/ (baseline lipids level) $* 100 \%$

All the statistical analysis above was performed using SPSS 22.0 (IBM Corporation, Armonk, New York, US). Multifactor-dimensionality reduction (MDR) method

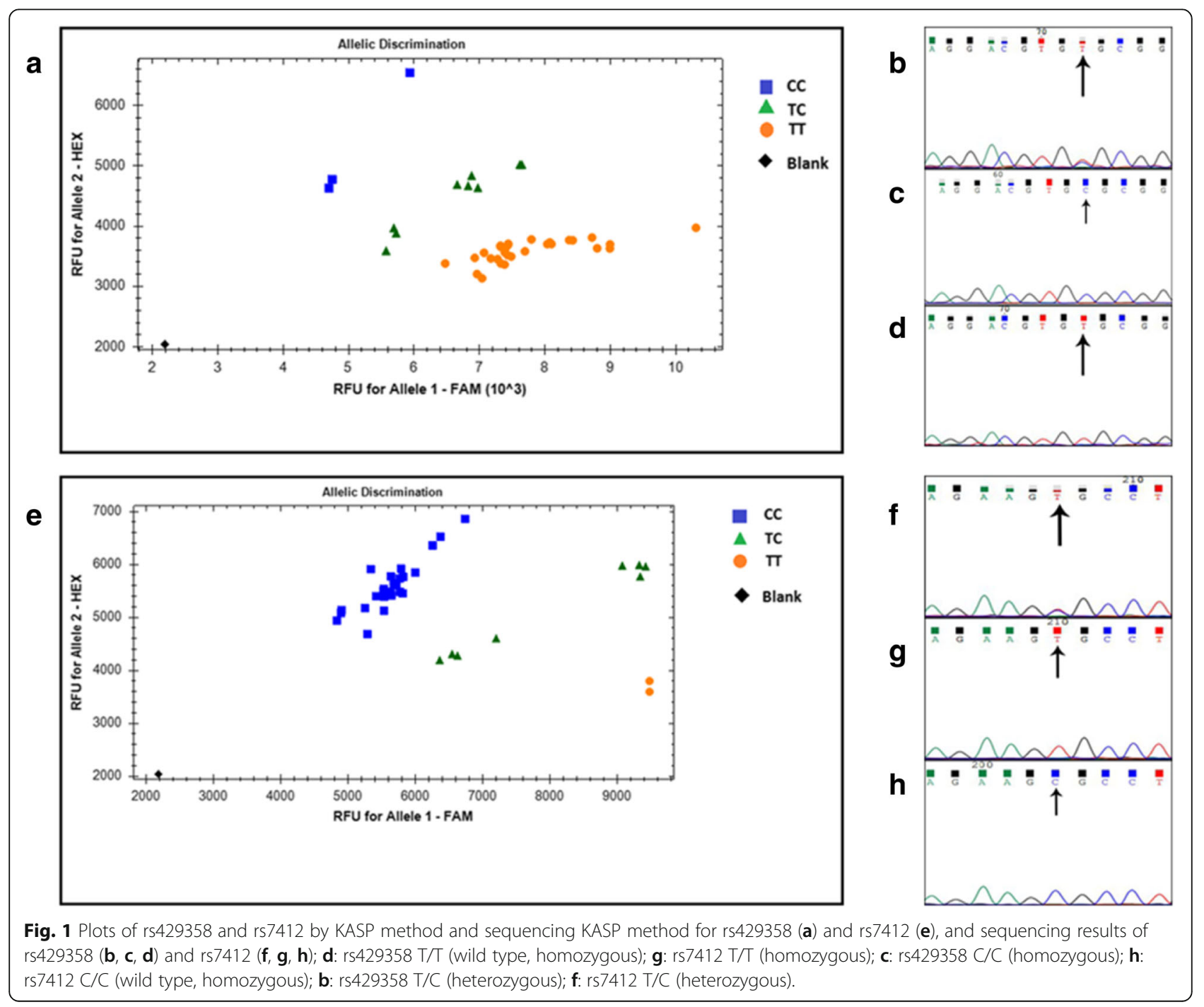


was performed to investigate gene-environmental interactions [26], such as interactions among genes, smoking, alcohol drinking, and T2DM. Best model with maximization of cross-validation consistency (CVC) was selected, and $P$ values of prediction accuracy were determined by 100 permutations. The $P$ values of less than 0.05 were considered statistically significant.

\section{Results}

\section{Clinical characteristics of patients}

The results of ApoE genotyping using KASP method or sequencing were consistent with each other (Fig. 1), indicating the KASP method was reliable and the ApoE genotyping results were convincing. The distribution of the ApoE genotypes in the total of 1002 individuals was shown (Table 3). The genotype e3/e3 was most frequent, and two genotypes (e2/e3, e3/e4) had a similar frequency.

\section{Lipid profile related to sex, diabetes and lifestyle}

Females have significantly higher levels in TC and HDL-C either in the baseline levels $(P=0.005,<0.001$, respectively; Fig. $2 \mathrm{a})$ or after statin treatment $(P<0.001$, both; Fig. 2b) than males. However, the lipid reduction percentage after statins treatment has no significant difference between men and women.

The TG and HDL-C baseline levels of dyslipidemia patients with T2DM were greater than the patients without diabetes $(P<0.001,=0.017$, respectively). And the two groups also had a significant difference after statin therapy in TG and HDL-C levels $(P=0.01,0.007$, respectively; Fig. $2 c, d)$. There was no significant lipid reduction percentage between the two statin drugs (atorvastatin/rosuvastatin).

Patients with smoking had a higher LDL-C baseline and a lower HDL-C baseline and lower elevation of HDL-C level after statins treatment than the non-smoking patients $(P=0.017,<0.001$ and $=0.026$ respectively; Fig.3a, $\mathrm{b}, \mathrm{c})$. And the cases with effective LDL-C lowering response, was statistically more in patients without smoking and alcohol drinking status compared with patients in smoking and alcohol drinking status (Fig. 3d, e).

\section{Statins response associated with ApoE genotypes}

Distributions of lipids profile in different ApoE isoforms and the response to statins were described (Table 4). A total of 1002 patients were divided into three groups by the ApoE isoforms: E2 (e2/e3, e2/e2), E3 (e3/e3, e2/e4), E4 (e3e4/, e4/e4) phenotype groups. The E2 group had the highest TG baseline, and there was a significant difference between E2 and E3 groups ( $P=0.026$, Fig. 4a). The E4 group had the highest LDL-C baseline, and the significant difference was found among E2, E3, and E4 $(P=0.007,0.005$, respectively, Fig. $4 \mathrm{~b})$. The reduce percentages of TG, TC, and LDL-C after statins treatment had a statistical difference among E2, E3, E4 groups, showing a significant decrease gradually from E2 to E3 and further to E4 groups (Fig. 5a, b, d). However, no significant difference was found in HDL-C reduction $(P>$ 0.05; Fig. 5c).

\section{MDR analysis of gene-lifestyle interaction}

One four-locus model rs7412-smoking-alcohol-drinking-T2DM had a maximum testing accuracy of 54.94\% and a maximum cross-validation consistency (100/100) that was significant $(P=0.047)$ after permutation testing (Table 5). The four-locus rs7412 combinations risk factors for each multilocus-factor combination were shown in Fig. 6. The combinations of rs7412 T/C-no smoking-no drinking-noT2DM had a high effect in LDL-C lowering; fewer patients with smoking or alcohol drinking achieved a better therapeutic effect when they carried rs7412 T/C or $\mathrm{C} / \mathrm{C}$ genotypes, such as patients in $\mathrm{E} 4$ groups. The models of rs429358-smoking-alcohol drinking-T2DM did not get a significant $P$-value after permutation test, even if the CVC was (100/100).

\section{Discussion}

In this study, we investigated the association between two common ApoE polymorphisms (rs7412 and rs429358) and baseline lipid profiles and statins response in dyslipidemia ASCVD patients classified with clinical E2/3/4 phenotypes.

As we are known, ApoE plays an important role in lipids clearance and lipoprotein metabolism [27]. The ApoE gene has three common alleles e2, e3 and e4 encoded three amino acid isoforms: ApoE2, E3 and E4, therefore, producing six common genotypes (e2/e2, e2/e3, e3/e4, e4/ 4, e3/e3, e2/e4.) [28]. ApoE4 isoform has a higher affinity for receptors of low-density lipoprotein receptor (LDLR) family, such as LDLR, very low-density lipoprotein (VLDL) and LDLR-related protein (LRP) and apoE receptor 2, which result in higher uptake or degradation of apoE. This would generate lower cholesterol efflux activity, which would produce elevated cholesterol levels in circulation and lead to a higher risk of cardiovascular diseases [27]. E2 amino acid isoform has a low affinity with VLDL, which induced an increased VLDL in plasma, that is why lots of patients with type III hyperlipidemia (HLP III) were

Table 3 Frequencies of ApoE genotypes and alleles in the study population

\begin{tabular}{llllllllll}
\hline Genotype & e2/e2 & e2/e3 & e2/e4 & e3/e3 & e3/e4 & e4/e4 & e2 & e3 & e4 \\
\hline Frequency (n) & 2 & 162 & 14 & 633 & 177 & 14 & 180 & 1605 & 219 \\
Percent (\%) & 0.2 & 16.2 & 1.4 & 63.2 & 17.6 & 1.4 & 8.98 & 80.09 & 10.93 \\
\hline
\end{tabular}


a



c

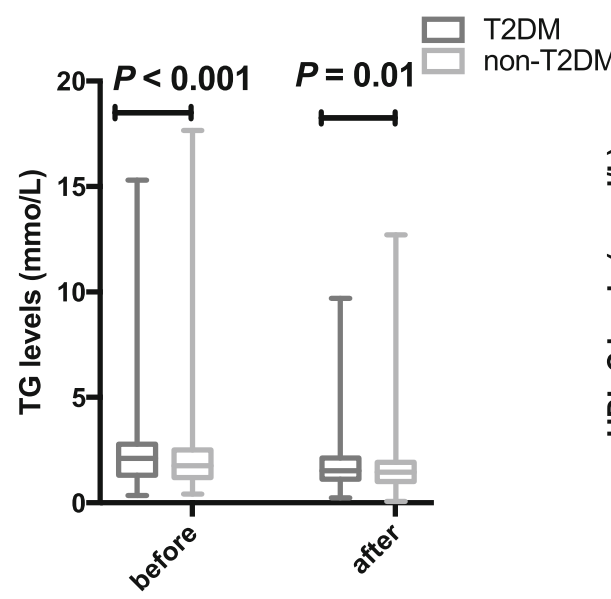

b



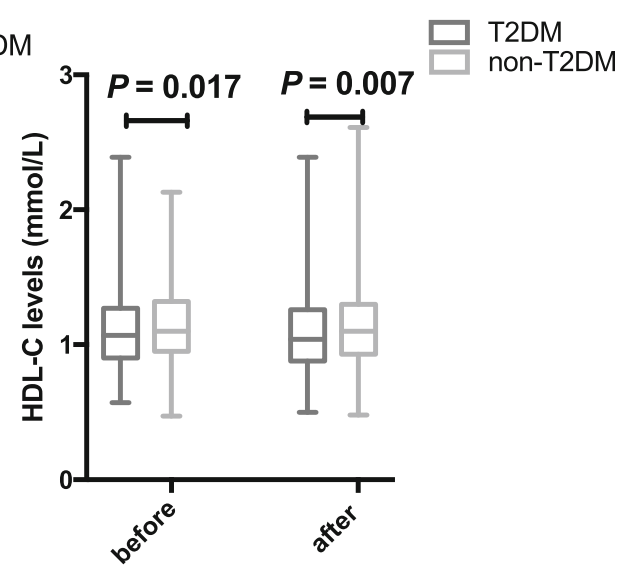

Fig. 2 Changes of lipids profiles with statin treatment The difference between male and female of statin therapy in TC (a) and LDL-C (b) levels. The difference between T2DM and non-T2DM of statin therapy in TG (c) and HDL-C (d) levels.

e2 homozygous [29]. However, people with e2 homozygotes got type III HLP less than 10\%, indicated that other genetic factors might be contributing to this disease in its progression [30].

We got some coincident results with the most previous study. First, TG baseline was higher in dyslipidemia patients with T2DM compared to those without T2DM [31]. Second, female patients had higher baselines of TC, LDL-C, and HDL-C. Third, there were significantly increased baselines of LDL-C from E2 to E3 and further to E4 groups.

Significant differences were also found in lipids response to statins among the three ApoE phenotypes in this study, E2 phenotype groups had a better lowering cholesterol effect than E4 phenotype groups. Although the HDL-C increment percentage among the three groups had no statistically significant difference, e2 carriers showed a better elevation. As a meta-analysis of statins response to ApoE polymorphisms that e4 carriers had a worse response to statin treatment than that of $\mathrm{e} 2$ carriers, mainly in
Caucasian [32]. Actually, Statins response to lipids associated with ApoE polymorphisms has been reported in several studies, but the results were inconsistent [17, 18, 32]. Ethnicities, therapy plan, statistical method selection and other genes associated with cholesterol biosynthesis and metabolism, might be possible causes of variation in response to statins. Since the dosage of statins in China is much lower compared that in the western country and Chinese might have a different response to statin therapy, the association study between ApoE polymorphisms and statin effects in Chinese is required.

Consistent with other investigations, the patients with smoking had a significantly higher baseline LDL-C levels and lower HDL-C levels than the non-smoking people and the non-smoking people also had a higher HDL-C level than the smokers after statins therapy. These discoveries may be revealing that smoking is a negative factor in lipid-lowing therapy of statins, which indicate lifestyles management combined with statin therapy is necessary. 



Fig. 3 Associations between lifestyles and statins responses The difference between smoking patients and non-smoking patients in LDL-C (a) and HDL-C (b) baseline levels. The difference in he HDL-C response to statins in smoking and non-smoking groups (c). Associations between lifestyles and statins responses to LDL-C. (d: smoking status, e: alcohol drinking status). "validness" means the LDL-C reduction percentage $\geq 25 \%$, and "voidness" means the LDL-C reduction percentage $<25 \%$.

Table 4 Distribution of lipids profile in different ApoE isoforms and the response to statins

\begin{tabular}{|c|c|c|c|c|}
\hline Lipid levels & $\mathrm{E} 2(n=164)$ & E3 $(n=647)$ & E4 $(n=191)$ & $P$ value \\
\hline \multicolumn{5}{|l|}{ before } \\
\hline $\mathrm{TG}$ & $2.14(1.30-3.00)$ & $1.83(1.24-2.48)$ & $1.81(1.24-2.68)$ & 0.031 \\
\hline $\mathrm{TC}$ & $4.80(4.31-5.44)$ & $4.87(4.22-5.68)$ & $5.01(4.27-5.69)$ & - \\
\hline $\mathrm{HDL}-\mathrm{C}$ & $1.06(0.92-1.32)$ & $1.09(0.94-1.31)$ & $1.11(0.94-1.27)$ & - \\
\hline LDL-C & $2.68(2.29-3.28)$ & $2.92(2.40-3.59)$ & $3.08(2.43-3.73)$ & 0.003 \\
\hline \multicolumn{5}{|l|}{ after } \\
\hline $\mathrm{TG}$ & $1.54(1.03-2.14)$ & $1.48(1.08-1.91)$ & $1.47(1.05-2.13)$ & - \\
\hline $\mathrm{TC}$ & $3.64(3.12-4.37)$ & $3.84(3.25-4.51)$ & $4.09(3.33-4.95)$ & 0.002 \\
\hline $\mathrm{HDL}-\mathrm{C}$ & $1.11(0.90-1.32)$ & $1.08(0.91-1.29)$ & $1.08(0.91-1.27)$ & - \\
\hline LDL-C & $1.89(1.50-2.35)$ & $2.08(1.68-2.68)$ & $2.25(1.76-2.87)$ & $<0.001$ \\
\hline \multicolumn{5}{|l|}{ reduction \% } \\
\hline $\mathrm{TG}$ & $25.20(5.30-41.57)$ & $21.03(-0.68-35.84)$ & $16.02(-4.17-33.33)$ & 0.021 \\
\hline TC & $23.56(10.73-31.24)$ & $20.49(10.70-30.47)$ & $17.92(5.20-28.92)$ & 0.012 \\
\hline $\mathrm{HDL}-\mathrm{C}$ & $-4.65(-17.56-10.58)$ & $0.85(-13.51-11.94)$ & $0(-12.94-11.38)$ & - \\
\hline LDL-C & $29.41(13.47-43.14)$ & $27.25(13.53-38.70)$ & $23.05(12.47-38.46)$ & 0.042 \\
\hline
\end{tabular}

Values are expressed as M (p25, p75); TC: total cholesterol; LDL-C: low-density lipoprotein cholesterol; HDL-C: high-density lipoprotein cholesterol; TG: triglycerides; $p$-values from K-W test, "-" means $P>0.05$. E2: e2/e2 and e2/e3, E3: e3/e3 and e2/e4, E4: e3/e4 and e4/e4; "before": before statin therapy; "after": after statin therapy 



Fig. 4 Lipids baseline levels among ApoE genotypes The association of ApoE polymorphisms and baseline levels in TG (a) and LDL-C (b).

According to our study, obviously different statins response to LDL-C in smoking and alcohol drinking habits was shown by different statistical methods. More patients with the SNP site rs7412 TC/CC in good living habits could achieve the therapy goal. And these results also indicate that medium dosage of statins to individuals with unhealthy lifestyles or underlying diseases might be ineffective in E4 patients.

In addition, today real-time quantitative PCR (q-PCR) and hybridization techniques were applied for ApoE genotyping in most clinic labs. However, KASP method established in our study has been verified using Sanger

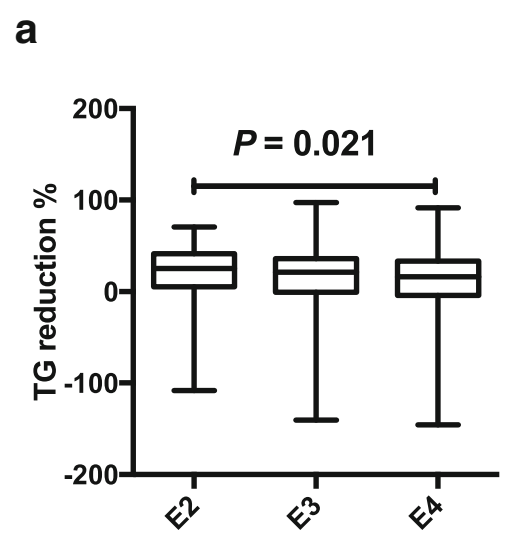

b

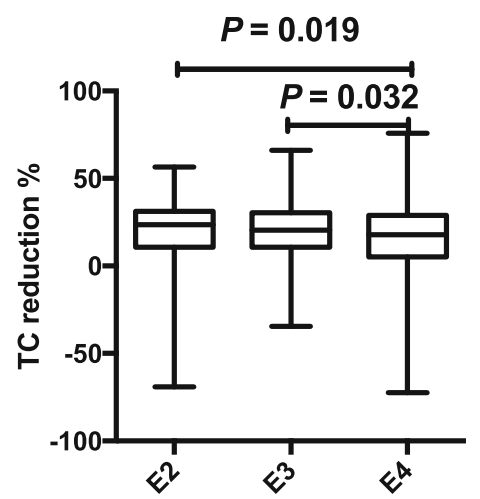

C

d

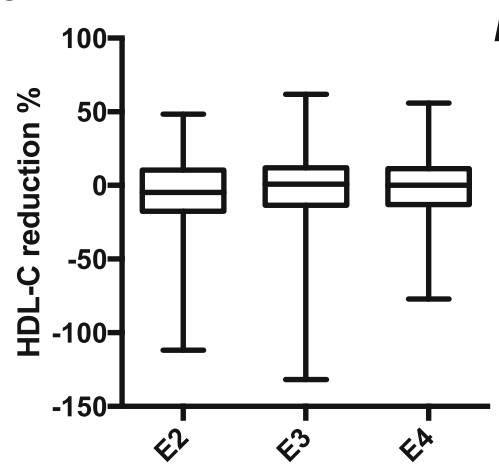

$P>0.05$



Fig. 5 Associations between statin response and ApoE variations The relationships between ApoE polymorphisms and lipids reduction with statin therapy in TG (a), TC (b), HDL-C (c) and LDL-C (d). 
Table 5 Multifactor dimensionality reduction analysis of rs7412 and smoking, alcohol drinking and T2DM

\begin{tabular}{llll}
\hline Model & Testing accuracy & CVC & $P$-value \\
\hline smoking & 0.505 & $90 / 100$ & 0.9241 \\
rs7412, smoking & 0.5492 & $100 / 100$ & 0.049 \\
rs7412, smoking, T2DM & 0.5174 & $82 / 100$ & 0.8132 \\
rs7412, smoking, alcohol drinking, T2DM & 0.5494 & $100 / 100$ & 0.047 \\
\hline
\end{tabular}

sequencing and was more economic compared to q-PCR methods while more convenient compared to hybridization methods $[19,33]$. The KASP tests could be finished in 1 PCR tube for 1 SNP after reaction results could be read directly with no more steps. Owing to less labeled-specific allele primers, the total price of the detections was costeffective in large-scale screening which was usual in research investigation. And this method has developed into a global benchmark technology in SNP genotyping platform [34], it would be acceptable for clinical diagnostic use in further medical research.

In this study, 1002 Chinese ASVCD patients with statin treatment in dysemia were recruited, it was a larger population study compared to other ethnicity studies previously $[17,18]$. Our research could be providing a reference for the clinical applications of statins to kinds of patients in drug selecting and individual-based treatment.

Some limitations of our study merit consideration. Firstly, due to the lack of the RNA samples, analysis the relationships between ApoE polymorphisms and its protein expression before and after statins therapy was not available. Secondly, exploring the mechanisms of the SNPs in the statins therapy would be necessary for further study. At last, the lipids regulation of statins was a complex process involved pharmacodynamics and pharmacokinetics with kinds of organs and genes, comprehensive furthermore exploration on gene panels is needed.

\section{Conclusions}

This study confirms the strong relationships between ApoE polymorphisms and lipids baseline levels, so as to the lipids responses to statins among ApoE protein isoforms in Chinese dyslipidemia ASCVD patients, and the self-build KASP ApoE genotyping method was reliable with great application prospect. Enhance drugs dose and better living habits management in ApoE4 phenotype patients could be more efficient in the clinical treatment in China.

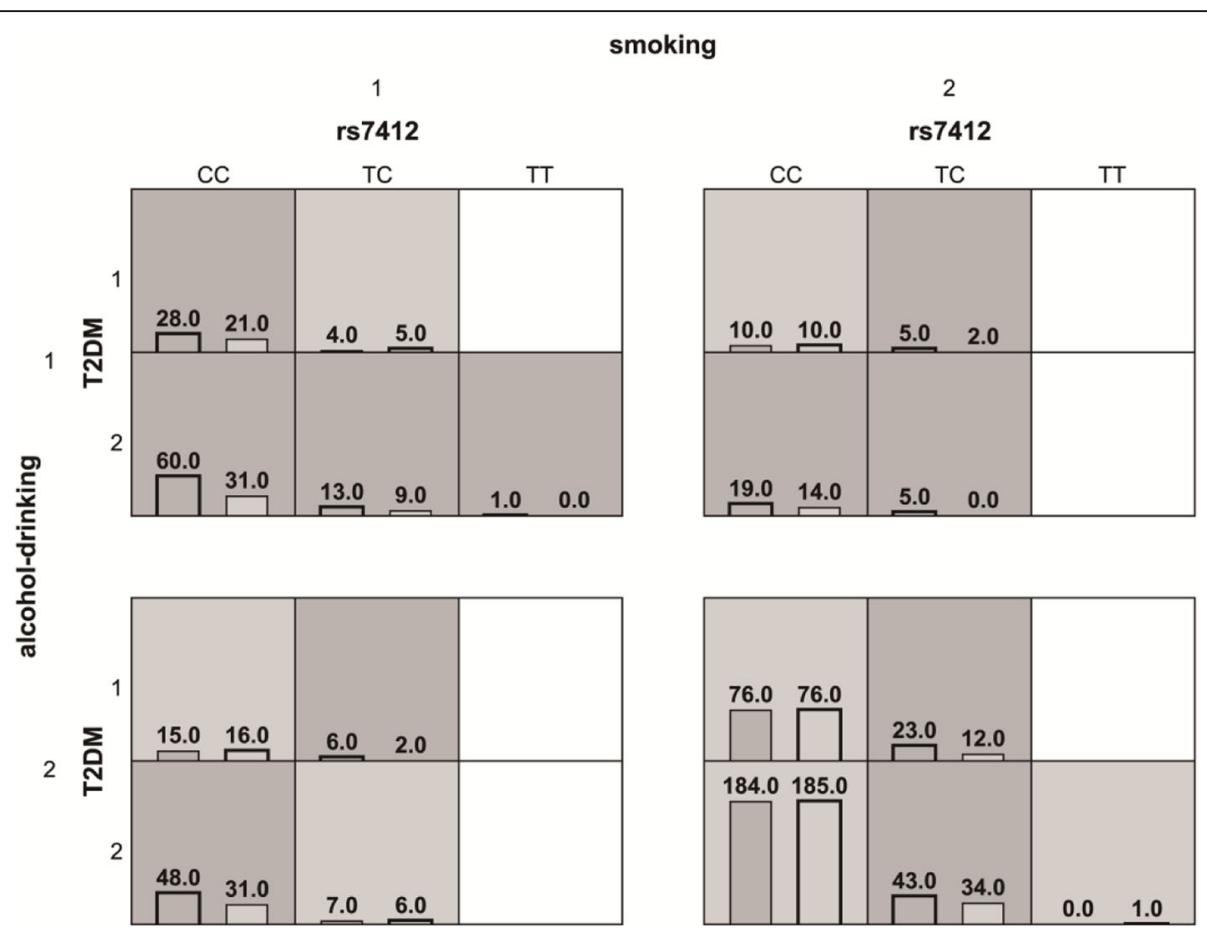

Fig. 6 Distribution of high-risk and low-risk genotypes in the best four-locus model Distribution of high-effective and low-effective models associated with statin response to LDL-C among rs7412, smoking status, alcohol drinking, and T2DM. The numbers of "effective" patients (left) and "non-effective" (right) are shown in cells. Dark gray indicates high-effective models, gray cells indicate low-effective models. Blank cells indicate no subjects. "1" means have the habit of smoking/drinking or the underlying disease of T2DM, "2" means negative of them. 


\section{Abbreviations}

ApoE: Apolipoprotein E; ASCVD: Arteriosclerotic cardiovascular diseas; CVC: cross-validation consistency; HDL-C: High-density lipoprotein cholesterol; HLP III: Type III hyperlipidemia; HRM: high resolution melting; KASP: Kompetitive Allele Specific PCR; LDL-C: Low-density lipoprotein cholesterol; MDR: Multifactor-dimensionality reduction; PCR: Polymerase chain reaction; Q-PCR: Real-time Quantitative PCR; SNP: single nucleotide polymorphism; T2DM: Type 2 diabetes mellitus; TC: Total cholesterol; TG: Triglyceride; VLDL: very low-density lipoprotein

\section{Acknowledgments}

The authors are very grateful to all participants.

\section{Funding}

This study was supported by grants from the National Nature Science Foundation of China (Grant No. 81270365), Hubei Province Health and Family Planning Scientific Research Project (Grant No. WJ2018H0013).

\section{Availability of data and materials}

All data generated or analyzed during this study are included in this published article and its supplementary information file.

\section{Authors' contributions}

FZ conceived the study, drafted the manuscript, reviewed and approved the final version of the manuscript. LZ and SH performed the molecular experiment, drafted the manuscript. LZ performed the KASP tests. LZ, SH, and $X G$ collected clinical data. $L Z$ and $S H$ performed the statistical analysis. $X C$, SL, and YN performed lipid profiling. All authors contributed to critically revised.

\section{Ethics approval and consent to participate}

The Ethics Committee of Zhongnan Hospital approved this study.

\section{Consent for publication}

Participants were informed of data sharing with their name and identity hidden per consent.

\section{Competing interests}

The authors declared no competing interests.

\section{Publisher's Note}

Springer Nature remains neutral with regard to jurisdictional claims in published maps and institutional affiliations.

\section{Author details}

${ }^{1}$ Center for Gene Diagnosis, Zhongnan Hospital of Wuhan University, Donghu Road 169\#, Wuhan 430071, China. ${ }^{2}$ Department of Cardiology, Zhongnan Hospital of Wuhan University, Donghu Road 169\#, Wuhan 430071, China.

Received: 29 August 2018 Accepted: 15 May 2019

Published online: 01 June 2019

\section{References}

1. Wang CY, Liu PY, Liao JK. Pleiotropic effects of statin therapy: molecular mechanisms and clinical results. Trends Mol Med. 2008;14(1):37-44.

2. Schmitz G, Langmann T. Pharmacogenomics of cholesterol-lowering therapy. Vasc Pharmacol. 2006;44(2):75-89.

3. Kajinami K, Akao H, Polisecki E, et al. Pharmacogenomics of statin responsiveness. Am J Cardiol. 2005;96(9):65-70

4. Lahoz C, Peña R, Mostaza JM, et al. Baseline levels of low-density lipoprotein cholesterol and lipoprotein (a) and the Avall polymorphism of the lowdensity lipoprotein receptor gene influence the response of low-density lipoprotein cholesterol to pravastatin treatment. Metabolism. 2005;54(6): $741-7$.

5. Willrich MA, Hirata MH, Genvigir FD, et al. CYP3A53A allele is associated with reduced lowering-lipid response to atorvastatin in individuals with hypercholesterolemia. Clin Chim Acta. 2008;398(1):15-20.

6. Singh PP, Singh M, Mastana SS. APOE distribution in world populations with new data from India and the UK. Ann Hum Biol. 2006;33(3):279-308.
7. Ferreira CN, Carvalho MG, Fernandes AP, et al. Comparative study of apolipoprotein-E polymorphism and plasma lipid levels in dyslipidemic and asymptomatic subjects, and their implication in cardio/cerebro-vascular disorders. Neurochem Int. 2010;56(1):177-82.

8. Grundy SM. Drug therapy of the metabolic syndrome: minimizing the emerging crisis in polypharmacy. Nat Rev Drug Discov. 2006;5(4):295-309.

9. Yousuf FA, Review IMP. Apolipoprotein E (Apo E) gene polymorphism and coronary heart disease in Asian populations. Pak J Pharm Sci. 2015;28:1439-44.

10. Chaudhary R. Apolipoprotein E gene polymorphism: effects on plasma lipids and risk of type 2 diabetes and coronary artery disease. Cardiovasc Diabetol 2012; 11(1): p. 36-36.

11. Fallah $\mathrm{S}$, Seifi M, Firoozrai M, et al. Effect of apolipoprotein E genotypes on incidence and development of coronary stenosis in Iranian patients with coronary artery disease. J Clin Lab Anal. 2011;25(1):43-6.

12. Kharrazi H, Vaisi RA, Sabokroh AR, et al. Association between apolipoprotein E polymorphism and coronary artery disease in the Kermanshah population in Iran. Clin Biochem. 2006;39(6):613-6.

13. Aydogan HY, Isbir S, Kurnaz O, et al. Associations of lipoprotein lipase S447X and apolipoprotein E genotypes with low-density lipoprotein subfractions in Turkish patients with coronary artery disease. Vivo. 2009;23(1):155-61.

14. Yilmaz-Aydogan $\mathrm{H}$, Kucukhuseyin $\mathrm{O}$, Kurnaz $\mathrm{O}$, et al. Investigation of polymorphic variants of PPARD and APOE genes in Turkish coronary heart disease patients. DNA Cell Biol. 2012;31(5):867-75.

15. Hagberg JM, Wilund KR, Ferrell RE. APO E gene and gene-environment effects on plasma lipoprotein-lipid levels. Physiol Genomics. 2000;4(2):101-8.

16. Rui B, Rebelo M, Decqmota J, et al. Apolipoprotein E epsilon-4 polymorphism is associated with younger age at referral to a lipidology clinic and a poorer response to lipid-lowering therapy. Lipids Health Dis 2011; 10(1): p. 48-48.

17. Lagos J, Zambrano T, Rosales A, et al. APOE polymorphisms contribute to reduced atorvastatin response in Chilean Amerindian subjects. Int J Mol Sci. 2015;16(4):7890-9.

18. Hu M, Mak WWL, Tomlinson B. Polymorphisms in apolipoprotein E and apolipoprotein $\mathrm{A}-\mathrm{V}$ do not influence the lipid response to rosuvastatin but are associated with baseline lipid levels in Chinese patients with hyperlipidemia. J Clin Lipidol. 2012;6(6):585-92.

19. Zhong $L$, Xie $Y$-Z, Cao T-T, et al. A rapid and cost-effective method for genotyping apolipoprotein E gene polymorphism. Mol Neurodegener. 2016; 11(1):2.

20. Zhan X-H, Zha G-C, Jiao J-W, et al. Rapid identification of apolipoprotein E genotypes by high-resolution melting analysis in Chinese Han and African fang populations. Exp Ther Med. 2015;9(2):469-75.

21. He C, Holme J, and Anthony J. SNP genotyping: the KASP assay, in Crop Breeding: Methods and Protocols, D Fleury and R Whitford, Editors 2014, Springer New York: New York, NY. p. 75-86.

22. Zhao SP. Key points and comments on the 2016 Chinese guideline for the management of dyslipidemia in adults. Zhonghua Xin Xue Guan Bing Za Zhi. 2016:44(10):827-9.

23. Catapano AL, Graham I, De Backer G, et al. 2016 ESC/EAS guidelines for the Management of Dyslipidaemias. Eur Heart J. 2016;37(39):2999-3058.

24. Peng C, Deng Q, Li Z, et al. Risk-association of DNMT1 gene polymorphisms with coronary artery disease in Chinese Han population. Int J Mol Sci. 2014; 15(12):22694-705.

25. Rabar S, Harker M, O'Flynn N, et al. Lipid modification and cardiovascular risk assessment for the primary and secondary prevention of cardiovascular disease: summary of updated NICE guidance. Bmj. 2014;349(349):g4356.

26. Ritchie $M$ D, Hahn $L W$, Roodi $N$, ., et al. Multifactor-dimensionality reduction reveals high-order interactions among estrogen-metabolism genes in sporadic breast cancer. Am J Hum Genet 2001; 69(1): p. 138-147.

27. Minihane AM, Jofre-Monseny L, Olano-Martin E, et al. ApoE genotype, cardiovascular risk and responsiveness to dietary fat manipulation. Proc Nutr Soc. 2007;66(2):183-97.

28. Nabatchian F, Khaghani S, Miri R, et al. Apolipoprotein E polymorphism, paraoxonase-1 activity and coronary artery disease: is there a link. Pak J Med Sci Q. 2008;24(24):204-8.

29. Song Y, Stampfer MJ, Liu S. Meta-analysis: apolipoprotein E genotypes and risk for coronary heart disease. Ann Intern Med. 2004;141(2):137-47.

30. Evans D, Seedorf U, Beil F. Polymorphisms in the apolipoprotein A5 (APOA5) gene and type III hyperlipidemia. Clin Genet. 2005;68(4):369-72.

31. Muhlestein JB, May HT, Jensen JR, et al. The reduction of inflammatory biomarkers by statin, fibrate, and combination therapy among diabetic 
patients with mixed dyslipidemia : the DIACOR (diabetes and combined lipid therapy regimen) study. J Am Coll Cardiol. 2006;48(2):396-401.

32. Zintzaras E, Kitsios GD, Triposkiadis F, et al. APOE gene polymorphisms and response to statin therapy. Pharmacogenomics J. 2009;9(4):248-57.

33. Richard P, Thomas G, de Zulueta MP, et al. Common and rare genotypes of human apolipoprotein $E$ determined by specific restriction profiles of polymerase chain reaction-amplified DNA. Clin Chem. 1994;40(1):24-9.

34. Ertiro BT, Ogugo V, Worku M, et al. Comparison of Kompetitive allele specific PCR (KASP) and genotyping by sequencing (GBS) for quality control analysis in maize. BMC Genomics. 2015;16:908

Ready to submit your research? Choose BMC and benefit from:

- fast, convenient online submission

- thorough peer review by experienced researchers in your field

- rapid publication on acceptance

- support for research data, including large and complex data types

- gold Open Access which fosters wider collaboration and increased citations

- maximum visibility for your research: over $100 \mathrm{M}$ website views per year

At $\mathrm{BMC}$, research is always in progress.

Learn more biomedcentral.com/submissions 